ISSN: 2600-5859

\title{
Estrategia pedagógica para el desarrollo de la competencia de autonomía emocional y moral en los estudiantes de Turismo de la Universidad de La Habana
} (c) $)$ (i) (9) (2)

Pedagogical strategy for the development of the competence of emotional and moral autonomy in the students of Tourism of the University of Havana

Msc. Yulima Daimet Valdés Bencomo. ${ }^{1}$ \& Dr.C: Orietta Martínez Chacón. ${ }^{2}$

Recibido: 06-12-2019 / Revisado: 07-12-2019 / Aceptado: 09-12-2019 / Publicado: 03-01-2020

Resumen.

DOI: https://doi.org/10.33262/concienciadigital.v3i1.995

El trabajo presenta la elaboración de la Estrategia Pedagógica para el desarrollo de la competencia de autonomía emocional y moral (EPDCAEM) en la formación profesional de estudiantes de turismo de la Universidad de La Habana, se fundamentó en el enfoque histórico cultural del desarrollo humano que sustenta la concepción de enseñanza aprendizaje desarrollador. Resultado que constituyó el objetivo del estudio. La estrategia metodológica seguida fue el enfoque mixto complejo en tres etapas: exploratoria, descriptiva transformadora y transformadora, mediante recursos de la Investigación Acción Participativa (IAP), en los ciclos: planeación, observación, reflexión acción. Se precisa el concepto de competencia de autonomía emocional y moral resultante de la sistematización teórica, al considerar indicadores emergentes desde su desarrollo, mediante procedimientos vigotskianos de diagnóstico actual y potencial, durante la construcción progresiva y verificación de la estrategia, con la participación activa de profesores y estudiantes, desde la disciplina principal integradora, en el proceso formativo. Se concluye sobre el valor del fundamento teórico metodológico asumido, la perspectiva holística e integradora de la EPDCAEM

\footnotetext{
${ }^{1}$ Universidad de la Habana. La Habana, Cuba. ybencomno@ftur.uh.cu

${ }^{2}$ Junta Acreditadora del MES. La Habana, Cuba. orymchacon@gmail.com
} 
del profesional de turismo y su carácter dinámico, flexible, funcional, transformador, transversal a la instrucción y educación en la formación, desde la ciencia pedagógica.

Palabras claves: Competencia de autonomía emocional y moral.

\section{Abstract.}

The work presents the elaboration of the Pedagogic Strategy for the development of the competition of emotional and moral autonomy (EPDCAEM) in the professional formation of students of tourism of the University of Havana, it was based in the cultural historical focus of the human development that sustains the conception of teaching learning developer. Result that it constituted the objective of the study. The followed methodological strategy was the complex mixed focus in three stages: exploratory, transform descriptive and transform, by means of resources of the Investigation Action Participative (IAP), in the cycles: planning, observation, reflection action. Is necessary the concept of competition of emotional autonomy and resulting morals of the theoretical systematizing, when considering emergent indicators from their development, by means of procedures vigotskianos of current and potential diagnosis, during the progressive construction and verification of the strategy, with the active participation of professors and students, from the integrative main discipline, in the formative process. You concludes on the value of the assumed methodological theoretical foundation, the holistic and integrative perspective of the professional's of tourism EPDCAEM and their dynamic, flexible, functional character, transformer, traverse to the instruction and education in the formation, from the pedagogic science.

Keywords: Competition of emotional and moral autonomy.

\section{Introducción.}

A partir del siglo XX se empieza a emplear el término estrategia en el contexto educativo Díaz (2009), así las estrategias pedagógicas se han abordado desde diferentes perspectivas. Autores como García y Elosúa (1993) consideran que las estrategias expresan la concepción y orientación fundamental de la formación y educación de la personalidad en su sentido individual, grupal y social, elemento importante a tener en cuenta en la presente investigación, así como el papel que ocupa en el sistema educacional. Otros autores como Sierra, R. (2004) pondera la estrategia como alternativa pedagógica en la dirección del proceso educativo.

La estrategia direcciona y organiza el hacer pedagógico a nivel institucional en función de sus objetivos formativos, para esta investigación, serán incluidos en los objetivos formativos el desarrollo de la competencia de autonomía emocional y moral. 
En cuanto a esta realidad es pertinente preguntarse por el quehacer del acto pedagógico, lo que implica pensar tanto en términos epistemológicos como funcionales y operativos. Por ende, se podría pensar en las estrategias pedagógicas que permitan implementar la intencionalidad del acto educativo en coherencia con el desarrollo de la competencia en cuestión.

Sierra (2008) puntualiza como elemento contextual de las estrategias pedagógicas los objetivos centrados en el desarrollo humano contenido estructural a considerar en la investigación.

Por otro lado, Vargas (2015) analiza la estrategia pedagógica como acciones del docente que facilitan el proceso de formación, tiene en cuenta el contexto, marco conceptual y postura teórica, lo que a juicio de la autora constituye el fundamento de una estrategia. Sosa, Añorga, Pichs, \& Rodríguez, (2016) la definen como arte para imaginar, organizar, dirigir con el ánimo de solucionar las contradicciones entre lo actuante y lo deseado. Moratalla (2005) considera el aspecto colaborativo, que para el presente estudio constituye un procedimiento que considera la relación intersubjetiva, a partir del EHC.

León (2012) hace énfasis en la planeación de objetivos, contenidos, métodos, medios y recursos, aunque flexibles, dinámicos y transformadores. Castellanos, et al (2002) concibe el carácter desarrollador de la estrategia, resaltando la transformación de la cultura y del sujeto, en lo que coinciden Martínez (2003), Frómeta R. (2007) y González (2009); estos autores consideran el proceso formativo en su relación con lo emocional, lo moral y la autonomía.

González.F. (2009), Alvarado, (2013), Nieva \& Martínez, (2016) ponderan la importancia del proceso educativo, donde los estudiantes y los profesores son sujetos protagónicos. Martínez $(2003,2013)$, destaca que el resultado del proceso de formación transcurre de manera planificada y organizada, además reflexiona sobre el papel activo e intencional del sujeto en el proceso de aprendizaje donde la valoración, autovaloración, la autonomía y autorregulación en la actividad y las tareas desarrolladoras constituyen elementos importantes para el logro de las metas propuesta. Estas premisas son consideradas para la elaboración de la estrategia pedagógica para el desarrollo de la competencia de autonomía emocional y moral.

Existe coincidencia entre los componentes que señalan autores como Martínez (2003, 2013), los que destacan los fundamentos que la sustentan, los principios que la regulan, los objetivos, métodos y procedimientos para alcanzar el fin propuesto desde su proyección, el proceso por el que transcurre, los recursos y la valoración sistemática de su resultado.

La estrategia para el desarrollo de la competencia de autonomía emocional y moral tiene como relevancia social que considera las necesidades e intereses, problemas y características 
de los estudiantes. Como fundamento pedagógico, es de vital importancia para el proceso de perfeccionamiento de la formación profesional mediante estrategias, el Enfoque Histórico Cultural de Vigotsky (1986), retomado por diversos autores como sustento de las estrategias pedagógicas, educativas y didáctica en sus investigaciones en la formación profesional y su materialización en la enseñanza aprendizaje desarrollador (Martínez, 2003; Castellanos, et al, 2002; Addine, 2004; Baquero, 2009; González F. , 2009; Nieva \& Martínez, 2017)

Se considera la interrelación dialógica docente estudiante Nouri ( 2014), la intersubjetividad, esencial en el proceso dialéctico de transformación (González F. , 2009; Martínez, 2014); la unidad de lo cognitivo y afectivo en las acciones de la estrategia pedagógica, que se manifiesta como un todo en el sujeto y constituye un principio básico para el desarrollo humano y la subjetividad. La contribución para la formación y el desarrollo integral de la personalidad, no solo supone fomentar debe tener en cuenta las diferencias individuales de los participantes y el contexto socioeconómico y cultural.

El diseño de las acciones en la estrategia, orientadas a los contenidos de desarrollo personal entre ellos: las dimensiones e indicadores de la competencia de autonomía emocional y moral, que manifiesten transformaciones en la actuación profesional (Martínez, 2003; Baquero, 2009; González F., 2009; Nieva \& Martínez, 2017).

La unidad de la actividad y la comunicación indispensable en la interacción de los sujetos entre sí y con el entorno social, permitiendo la transformación de estos y como principio mediatizador del desarrollo y autotransformación del sujeto en la actividad específica.

De acuerdo con lo expresado por los diversos autores, se asume que la estrategia pedagógica tiene componentes estructurales, a saber: principios que la regulan, una planeación o proyección que le da perspectiva, objetivos que la intencionan, procesos por los que transcurre, contextos a los que responde, metodología que la hace sistemática y la organiza, tareas desarrolladoras que la mediatizan.

Los componentes funcionales que la dinamizan están referidos a: los diagnósticos desde la ZDP, la mediación cultural, la dialéctica de la unidad cognitiva afectiva, la mediación intersubjetiva dada en la colaboración e interrelación dialógica, las transformaciones progresivas de los sujetos y de la práctica educativa y la formación Vigotsky (1987)

Los principios constituyen un componente esencial que guía y regula la estrategia, por lo que se debe reflejar en la materialización del resto de los componentes y en el resultado que esta tenga. Se asumen del análisis realizado del EHC, el carácter activo del sujeto en el aprendizaje, el carácter desarrollador del aprendizaje, el tránsito del aprendizaje de la intersubjetividad a la intrasubjetividad, el aprendizaje desde la potencialidad del desarrollo. 
Dada las particularidades de la Carrera de Turismo se consideran fundamentales para el diseño y elaboración de la estrategia pedagógica para el desarrollo de la competencia de autonomía emocional y moral, características esenciales como: la flexibilidad, intencionalidad, desarrolladora y transformadora de la realidad social, la vinculación a las esferas de formación profesional y adecuación de las formas organizativas a las especificidades de los estudiantes de turismo.

La estrategia pedagógica ha sido referida desde perspectivas diversas: como concepción, como sistema de actividades o como proceso. Para la autora de esta investigación, se asume como proceso y sistema de actividades pedagógicas que transcurre en el proceso formación profesional, considera las exigencias de la práctica profesional y el nivel de desarrollo de los sujetos.

Como proceso porque considera los niveles de desarrollo del sujeto en formación y su rol activo se dinamiza de lo simple a lo complejo, potencializa nuevos niveles de forma progresiva como sistema de actividades que se organizan de forma sistemática y progresiva según los resultados de los diagnósticos actuales (DA) y potenciales (DP) de los sujetos, incluye tareas pedagógicas que integran la institución y educación.

El diseño de la estrategia considera componentes estructurales y dinámicos funcionales en una relación dialéctica, que se materializa durante el desarrollo del proceso formativo, los que han sido identificados en la teoría y abarca en sentido general:

Componentes estructurales:

- Los principios que regulan, sistematizan, organizan, gradúan y controlan el desarrollo personal

- Los objetivos.

- Las fases o etapas

- El diagnóstico actual y diagnóstico potencial como procedimiento metodológico básico.

- Las tareas pedagógicas

- La necesidad de la formación profesional de los estudiantes y su desarrollo personal

Como componentes funcionales:

- El estudiante como sujeto de auto transformación y transformación.

- La unidad cognitiva afectiva y actividad comunicación.

- El contexto socio cultural de la profesión y el aprendizaje

- Su perspectiva desarrolladora

- Los componentes de autonomía emocional y moral, sus dimensiones: automotivación, responsabilidad, análisis crítico de las normas sociales, 
autocontrol, manejo de las relaciones, valores morales, comportamiento y decisión moral, socialización de las normas deberes y derechos, juicio moral, reflexividad moral, participación y empatía dialógica.

La estrategia pedagógica para los fines propuestos ha de incluir la organización del proceso de instrucción educación en la formación, teniendo en cuenta el papel de la disciplina principal integradora y el vínculo teoría practica en la integración del individuo a la sociedad, la concepción pedagógica integradora y su concreción en el proceso de enseñanza aprendizaje desarrollador.

\section{Desarrollo.}

La estrategia pedagógica para el desarrollo de la autonomía emocional y moral desde su conceptualización y los recursos de la IAP, articulado al proceso pedagógico, a través de las tareas pedagógicas.

Se revela en la reflexión de los profesores para la aplicación y verificación de la tarea pedagógica (situaciones) un cambio de concepción sobre el aprendizaje de los estudiantes y de los procedimientos metodológicos del proceso, así como su alcance a nivel de toda la formación.

Esta valoración fue apareciendo paulatinamente en la construcción de la estrategia, a partir de lo planteado por los profesores de las asignaturas de la disciplina principal integradora, desde las tareas pedagógicas y vivenciadas durante el proceso formativo, que constituyó eje dinamizador en el momento y etapa transformadora. Se establecieron como componentes estructural y funcional que la conforman: principios generales que la regulan, requisitos que la condicionan, objetivos que la intencionan, fases y procesos por los que transcurre, contextos a los que responde, las tareas pedagógicas como desarrolladoras que a la vez conforman el componente estructural eje que dinamiza el desarrollo de CAEM y la estrategia en todo su accionar.

Los componentes posibilitan que la estrategia transcienda al ámbito formativo en el vínculo de la instrucción y educación, se desarrolla a lo largo de todo el proceso de formación profesional, específicamente en la DPI y las asignaturas de la especialidad, aunque incluye e integra al resto de estas, bajo los fundamentos del aprendizaje desarrollador.

Las tareas abarcan el diagnostico actual y potencial, mediante criterios sobre la manifestación de las dimensiones y los indicadores de la competencia de autonomía emocional y moral como unidad dialéctica, propiciadoras de su desarrollo, son flexibles, se dirigen a los fines propuestos, desarrolladoras y transformadoras de la actuación en la realidad social, vincula la formación profesional, se adecuan a las formas organizativas y a las particularidades de los estudiantes.

Como resultado de este proceso se elaboró la estrategia pedagógica para el desarrollo de la competencia de autonomía emocional y moral, que se describió en párrafos anteriores, se 
representa a continuación en la figura 1, que muestra los componentes estructurales y funcionales para su implementación en el proceso de formación donde fue verificada. Estos son:

Figura N 1. La estrategia pedagógica para el desarrollo de la competencia de autonomía emocional y moral.

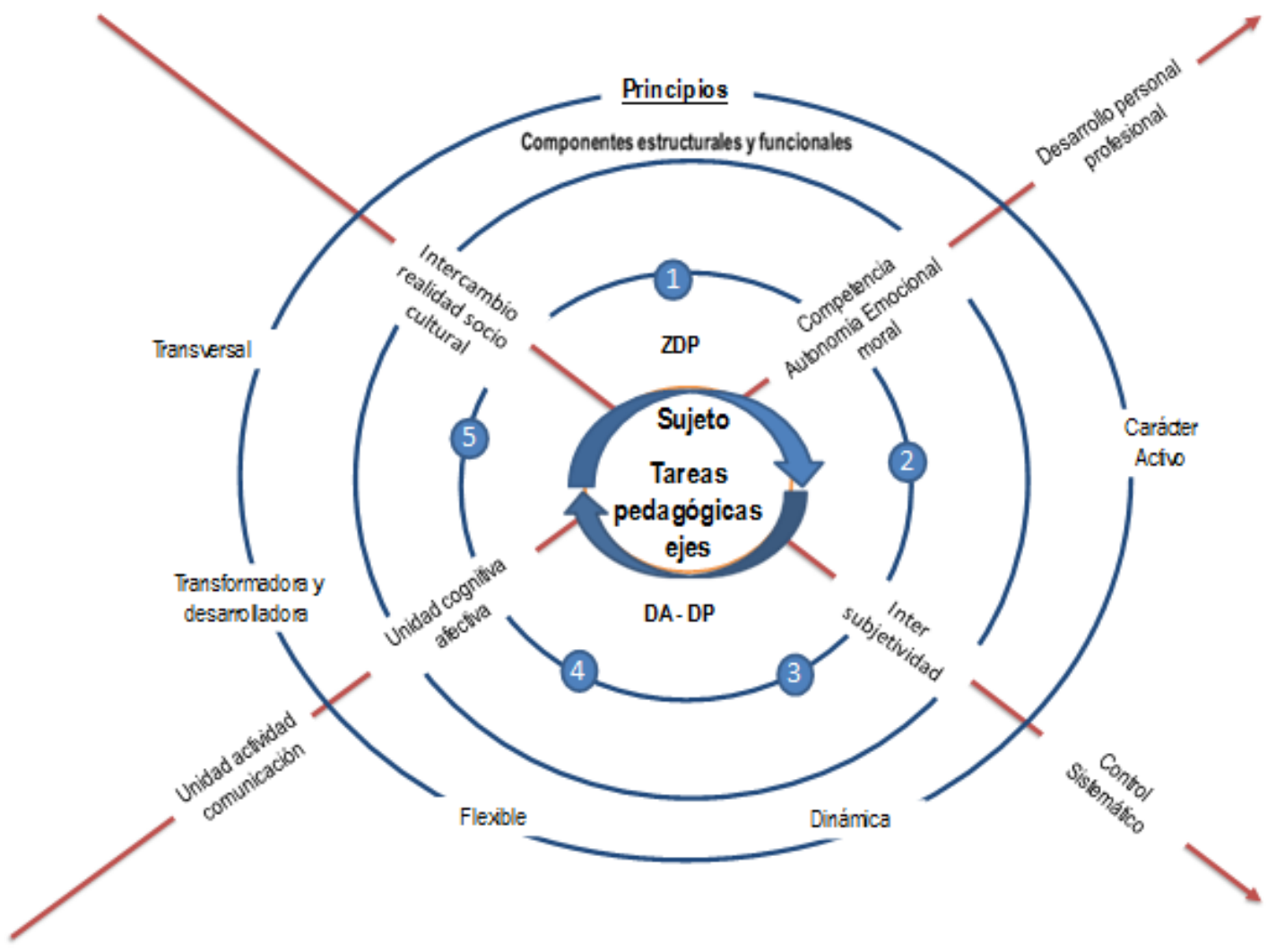

Fuente. Elaboración propia

Objetivo: Desarrollar la competencia de autonomía emocional y moral en los estudiantes de turismo, a partir de la implementación sistemática e intencionada en el proceso de formación del profesional de turismo.

Componentes estructurales de la estrategia pedagógica: principios, requisitos, características, fases por las que transcurre, la proyección y planificación, los componentes didácticos que abarcan los objetivos, contenidos, métodos, medios, el control y la evaluación, atendiendo a las asignaturas de la disciplina de la especialidad que son base de los objetivos formativos y su concreción en el proceso de enseñanza aprendizaje, así como los resultados de la estrategia pedagógica que considera su perfeccionamiento, el de las tareas ejes y el nivel de desarrollo de la competencia de autonomía emocional y moral en los estudiantes a partir de sus dimensiones e indicadores.

Los principios que se asumen son: 
ISSN: 2600-5859

1. La unidad dialéctica entre el componente afectivo emocional y moral con proyección social.

2. La unidad de la actividad y la comunicación como mediadora del aprendizaje para el desempeño profesional.

3. El vínculo entre la instrucción y la educación.

4. Transversal al proceso de formación de manera horizontal y vertical.

5. El rol activo, consciente y comprometido de los actores educativos en el proceso de formación y desarrollo de la competencia.

6. La educación como transformadora de la cultura y de los sujetos de aprendizaje.

7. El autoeducación y la auto transformación en el proceso de enseñanza aprendizaje desarrollador.

8. La unidad dialéctica de la competencia de autonomía emocional y moral y su manifestación futura en la actuación profesional.

Los principios descritos se concretan en requisitos a cumplir para el desarrollo del sistema de actividades, los que exigen:

1. El diagnóstico actual y potencial del nivel de desarrollo de la competencia de autonomía emocional y moral de los sujetos.

2. La elaboración de acciones sistemáticas y graduales, en correspondencia con los logros y limitaciones detectadas en los diagnósticos donde los sujetos son activos y autovalorativos.

3. La transcendencia al ámbito universitario insertándose a las esferas de formación profesional.

4. La adecuación a las formas organizativas y particularidades de los estudiantes de turismo y a las exigencias sociales y profesionales.

5. La concreción de la estrategia pedagógica en la disciplina integradora como vía para su implementación.

6. La modelación de la competencia de autonomía emocional y moral y las exigencias de la profesión en diversos contextos de actuación futura en el proceso de aprendizaje desarrollador.

7. Las tareas pedagógicas constituyen ejes con carácter integrador que dinamiza el proceso formativo.

8. La colaboración, el trabajo en equipo y el intercambio dialógico en la actividad formativa.

Las tareas pedagógicas se caracterizan por:

- La contribución al desarrollo de la competencia de autonomía emocional y moral.

- La flexibilidad, la intencionalidad, desarrolladoras y transformadoras del sujeto y su actividad en la realidad social.

- Las posibles soluciones no deben ser predeterminadas, son inestructuradas deben tener presencia de obstáculos 
- Movilizadoras de cuestionamientos, la problematización y elaboración de nuevas adecuaciones

- Propiciadoras de toma de decisiones morales a partir de la problematización, el manejo de alternativas de solución, con resultados posibles, consecuencias, así como la manifestación en la actuación de las normas profesionales personalizadas de manera responsable.

- El establecimiento del vínculo entre condiciones y exigencias individuales de la práctica profesional y social general.

- La consideración de lo perspectivo, pero estar próximas a la realidad.

- La modelación de la actividad profesional o salida de ella incluyendo elementos nuevos, cuyos resultados y efectividad dependen de las nuevas combinaciones que el sujeto pueda establecer de acuerdo a la asunción de las normas.

- La proyección de sistema de conductas alternativas ante diversas situaciones inesperadas de la realidad y la comunicación cumpliendo las reglas de la escucha activa que propicien su desarrollo.

- La exigencia de valoraciones, auto valoraciones, autonomía y auto regulación en la actividad real y futura.

Las tareas se relacionan con la actividad profesional, la modelan e incluyen elementos nuevos, no vistos con anterioridad, cuyos resultados y efectividad dependen de las nuevas combinaciones que el sujeto pueda establecer, de la previsión del resultado, de sus consecuencias, la valoración que determina qué debe hacer y cómo hacerlo, implicándose en el proceso de su realización, transformando las condiciones o los procedimientos para el logro del objetivo o modificándose a sí mismo. La tarea incluye el descubrimiento y la búsqueda de la solución, acorde con las exigencias que planteen. Martínez, (2010).

Fases de la Estrategia Pedagógica para el desarrollo de la competencia de autonomía emocional y Moral.

Fase 1: Orientación:

Objetivo de la fase: valorar la concepción de la CAEM, las posibilidades de su desarrollo mediante la EPDCAEM y las perspectivas de su implementación por los actores educativos implicados en la formación profesional de los estudiantes de turismo.

Descripción de la fase: consiste en la sensibilización de los actores educativos, estudiantes, y profesores de la disciplina principal integradora de la importancia y necesidad de desarrollar la competencia de autonomía emocional y moral (CAEM), desde su concepción mediante la estrategia pedagógica para el desarrollo de la CAEM, los procedimientos para su implementación, así como la elaboración de las tareas pedagógicas desarrolladoras (eje de la estrategia), por parte de los profesores, actores que intencionan el desarrollo de la CAEM de manera planificada y organizada.

En esta etapa es fundamental que los actores educativos se orienten en el proceso formativo de la CAEM y la concreción de su desarrollo en la actividad de aprendizaje, dado que, desde 
el EHC, el aprendizaje esta mediatizado primero en la dimensión afectiva, donde lo emocional da sentido positivo personalizado a los valores morales de la profesión y la sociedad, transitando del plano intersubjetivo al intrasubjetivo, que se manifiesta en el modo de actuación.

Acciones de la fase:

Conciliar de manera precisa los criterios sobre la necesidad e importancia del desarrollo de la CAEM en el proceso de formación profesional en los diversos años, por estudiantes y profesores.

Constatar el nivel de desarrollo de la CAEM, a partir de la aplicación del test de autonomía emocional y moral a los estudiantes al inicio del año, vía online, intercambiando sobre los resultados generales y la importancia para la actividad profesional.

Reflexionar por los profesores sobre los conceptos de CAEM; estrategia pedagógica para su desarrollo y tarea pedagógica desarrolladora, los requisitos para su elaboración y los procedimientos de aplicación de la EPDCAEM, valorando las posibilidades que brindan las disciplinas de la especialidad para su integración al PEA y las perspectivas de transformación social y profesional.

Diseñar las tareas pedagógicas eje de la estrategia, a partir de la consideración de sus requisitos y características, que modelan disyuntivas presentes en situaciones profesionales de diversas esferas de la actividad turística, para el diagnóstico y desarrollo de la CAEM, Fase 2. Proyección:

Objetivo de la fase: Programar las acciones de la estrategia pedagógica para el desarrollo de la CAEM considerando sus componentes estructurales y funcionales en la formación profesional de los estudiantes de turismo con perspectiva de integración al proceso de enseñanza aprendizaje, a partir del perfeccionamiento de las tareas pedagógicas diseñadas, mediante el intercambio científico pedagógico.

Descripción de la fase:

De acuerdo a los resultados de la fase de sensibilización que abarca el nivel de desarrollo de la CAEM, mediante el test y la actividad orientadora de los profesores, se perfeccionan las tareas pedagógicas y precisan los instrumentos que permitan la implementación posterior en el proceso formativo, mediante la disciplina y que contribuye al desarrollo humano de los profesionales en formación.

Confrontar los resultados con intencionalidad pedagógica y transformar la realidad educativa, de acuerdo a las demandas de la formación, del contexto actual y perspectivo del turismo y la sociedad.

La fase concibe el perfeccionamiento de las tareas pedagógicas diseñadas previamente, a partir de las siguientes consideraciones:

La modelación de situaciones de la práctica profesional en las que están presentes dilemas morales en diversas esferas de actuación; la inclusión de elementos esenciales que plasman los indicadores de la competencia de autonomía emocional y moral; demandan alternativas 
de solución e implican asumir posiciones de manera autónoma con implicación emocional y moral; permiten evidenciar el nivel de desarrollo en el que se encuentran los estudiantes (DA) y a su vez, las potencialidades de su desarrollo en el proceso formativo (DP), mediante la actividad reflexiva en diada o grupal; se plantean en diferentes niveles de complejidad que permitan la valoración del nivel de desarrollo y a la vez, valorar el progreso de los estudiantes en la medida que participan de su autotransformación.

Asimismo, incluye la determinación del estado de los recursos y materiales necesarios para la aplicación de la EPDCAEM, el control sistemático y final de la CAEM, así como las vías y actividades para el perfeccionamiento o adecuación de la estrategia.

Acciones de la fase:

Intercambiar sobre los niveles de desarrollo de la CAEM, constatados mediante el test de autonomía emocional y moral con la participación de los estudiantes y profesores

Determinar las condiciones educativas necesarias para el desarrollo del proceso de formación profesional, que posibilite el desarrollo de la CAEM en los estudiantes de la carrera de turismo.

Perfeccionar las tareas pedagógicas para el diagnóstico y desarrollo de la CAEM, a partir del intercambio y valoración científico pedagógico de la presencia en ellas de sus requisitos y características, los indicadores de las dimensiones de la CAEM, la modelación de situaciones disyuntivas o de dilemas de la realidad de la práctica profesional, los niveles de complejidad. Precisar los procederes generales para la aplicación de la EPDCAEM, a partir de su estado actual, para el control sistemático de progreso y final de la CAEM, así como las vías y actividades para el perfeccionamiento o adecuación de la estrategia.

Modelar la implementación de la estrategia pedagógica en el proceso formativo desde las disciplinas integradoras y los procedimientos para la implicación de todos los actores educativos que intencionan la formación en la carrera.

Proyectar los diagnósticos actuales y potenciales, así como los progresivos, mediante la tarea pedagógica y desarrolladora, el auto reporte y reporte como instrumento para la autovaloración y valoración con los otros.

Organizar las acciones de control sistemático y final del nivel de desarrollo de la CAEM y de la estrategia pedagógica para el desarrollo de esta.

Concertar un cronograma para la implementación de la EPDCAEM y presentación de sus resultados en actividades científicas pedagógicas a nivel de departamentos y la carrera Fase 3. Implementación de la EPDCAEM:

Objetivo de la fase: Desarrollar la competencia de autonomía emocional y moral en el proceso de formación del profesional de turismo, mediante la integración de la estrategia pedagógica al PEA desarrollador, especialmente desde la disciplina principal integradora y de la especialidad profesional, a partir del DA y DP que demanda la concepción de zona de desarrollo próximo del EHC, mediante la tarea pedagógica eje de la estrategia. 
Descripción de la fase: La implementación de la EPDCAEM integrada al proceso de formación y su materialización en el PEA desarrollador, inicia con la aplicación del DA y DP mediante la tarea pedagógica, que modela la práctica profesional verificado por Martínez (2003) y los dilemas que ella presenta, a través de los procedimientos descritos por Vigotsky de trabajo a solas y en dúo, a lo que se agrega la reflexión grupal.

Los estudiantes son considerados activos de su propia autoeducación y transformación desde los diagnósticos, cuyos resultados son asumidos como momentos del desarrollo personal por los que transita la CAEM en el proceso de formación, a partir de la concepción del EHC y de enseñanza aprendizaje desarrollador.

Las valoraciones de los resultados diagnósticos se apoyan, en esta fase, de un instrumento de auto reporte y reporte de los niveles de desarrollo manifestados en los resultados de las tareas, atendiendo a los indicadores de la CAEM, los que deben ser comparados por los estudiantes y reflexionados en el grupo, contrastado con las alternativas de solución, así como la correspondiente valoración del modo de actuación asumido en ellas.

La reflexión abarca las perspectivas de transformación propia y del futuro desempeño en la actividad profesional, que se da en los planos intersubjetivos hasta transitar al intrasubjetivo, donde se requiere una posición crítica, juicios de valor sobre la actuación, así como el sentido personalizado que se le atribuye, a partir de la valoración de las consecuencias de asumir una u otra posición en la solución de los dilemas que se presentan.

Desde esta perspectiva transformadora que propicia la tarea pedagógica, es que se le atribuye el papel eje en la estrategia, desarrollador de la competencia, a partir de su diseño y perfeccionamiento, así como su materialización en el proceso de formación profesional desde el EHC. La relación de la teoría con la práctica adquiere un sentido fundamental, en tanto las tareas son salidas de la práctica y la modelan, al igual que la CAEM. Además, sus resultados manifiestan las particularidades individuales de los estudiantes y comprometen su personalidad de manera integral, propiciando su desarrollo.

De igual modo se va confrontando no solo el accionar futuro de la actuación profesional en términos de habilidad, sino la autonomía emocional y moral que este manifiesta, esencial en el futuro desempeño y en correspondencia con los valores del proyecto social cubano.

Asimismo, en el proceso formativo de los estudiantes se verifican permanentemente las fases anteriores para hacer los ajustes conforme al progreso de los estudiantes, a las interacciones con sus compañeros y profesores, a partir de las soluciones y reflexiones realizadas, así como de los niveles de desarrollo alcanzados progresivamente.

Acciones de la fase:

Diagnosticar el nivel de desarrollo actual y potencial de la CAEM en los estudiantes con empleo del procedimiento a solas y en dúo respectivamente, mediante la tarea pedagógica que posee menor nivel de complejidad.

Valorar el nivel de desarrollo de la CAEM a partir de las soluciones dadas a las situaciones pedagógicas, mediante el auto reporte. 
Contrastar los resultados de las tareas pedagógicas en dúo, a partir del intercambio que posibilita el planteamiento de nuevas soluciones alternativas y la valoración de las soluciones del otro, mediante el reporte para su posterior contrastación.

Reflexionar en grupo sobre las soluciones a las situaciones pedagógicas, la manifestación de la autonomía emocional y moral, así como el nivel de desarrollo de la competencia.

Fase 4. Verificación

Objetivo de la fase: Verificar la EPDCAEM y el progreso de niveles de desarrollo de la CAEM.

Descripción de la fase: en esta se verifican las fases de orientación, proyección e implementación. Se reflexiona en actividad de los profesores, mediante taller, las dificultades presentadas, los problemas comunes y las alternativas de solución, así como los niveles de desarrollo de la CAEM expresados en el actuar por los estudiantes.

Acciones de la fase:

- Analizar los resultados alcanzados en los test y compararlos.

- Verificar los DA y DP por los estudiantes para auto valorar los niveles de progreso.

- Valorar el resultado de los diferentes reportes y auto reportes contrastando la autovaloración y la valoración como parte del desarrollo personal

- Verificar la estrategia pedagógica, sus componentes estructurales y funcionales, así como su carácter transversal a la formación.

Fase 5: Perfeccionamiento:

Objetivo de la fase: Adecuar la EPDCAEM, considerando los resultados obtenidos en las etapas de anteriores, considerando las nuevas exigencias del contexto sociocultural especifico.

Descripción de la fase: se valoran los resultados de las fases anteriores y las nuevas demandas que plantee la profesión y el contexto socio económico y cultural.

Se diseñan acciones de mejoras a partir de la valoración de los componentes estructurales y funcionales, así como del nivel de desarrollo de la competencia de autonomía emocional y moral en los estudiantes.

Se realizan los ajustes de EPDCAEM integrando a ella las acciones diseñadas. Acciones de la fase:

- Retroalimentar a los actores educativos sobre los resultados de la implementación de la estrategia.

- Evaluar la estrategia en los colectivos de disciplina y los años como parte del trabajo metodológico

- Realizar talleres científicos metodológicos a nivel de carrera como parte del perfeccionamiento de la formación profesional a nivel vertical.

- Organizar un nuevo ciclo de desarrollo de la CAEM, mediante la estrategia pedagógica. 
Una vez precisado los componentes estructurales es menester abordar los componentes dinámicos funcionales que posibilitan la expresión de las características esenciales como su flexibilidad, contextualización, transformadora de la realidad educativa y de la formación del futuro profesional del turismo.

Estos componentes son: los actores del aprendizaje y desarrollo de la competencia, la relación que se establece entre estos en el proceso de formación y durante la implementación de la estrategia, la mediación de la comunicación que dinamiza el proceso de aprendizaje y desarrollo de la competencia, la actividad en colaboración entre pares y la grupal, los diagnósticos actuales y potenciales que reflejan el progreso sistemático, la dinámica que propician las tareas ejes desarrolladoras que abarcan los diagnósticos, los procedimientos de valoración y autovaloración a partir de los indicadores de la competencia, los contextos que pueden reflejarse en la educación, las disyuntivas de las diversas situaciones que modelan la práctica profesional de turismo en la variedad de esferas de actuación y a la propia competencia a formar, así como las demandas y exigencias de los escenarios socio económico e histórico de la realidad cubana en los que se insertan los estudiantes presentes en el proceso formativo.

En el cierre del ciclo de la IAP: reflexión acción planeación reflexión, en el último taller se verificaron los componentes estructurales y dinámicos funcionales de la EPDCAEM, según los resultados y progresos de los estudiantes en el nivel de desarrollo de la competencia y se hicieron las precisiones y adecuaciones necesarias que dieron lugar a la elaboración final de la estrategia que ha sido expuesta.

\section{Conclusiones.}

- La implementación de la EPDCAEM posibilito el desarrollo de dicha competencia, a partir de los procedimientos diseñados que integran las tareas pedagógicas, los diagnósticos (DA, DP), la interrelación entre los implicados, así como los mecanismos autovalorativos y valorativos, desde el intercambio dialógico.

- Los componentes estructurales y funcionales de la EPCAEM se interrelacionan y permite la flexibilidad desde las proyecciones y transformaciones personales, sociales y profesionales en el proceso de formación profesional, así como la verificación del desarrollo personal y social desde lo intersubjetivo a lo intrasubjetivo.

- La EPCAEM verificada y contrastada en el PEA desarrollador con estudiantes se presenta como una alternativa válida para desarrollar la competencia de autonomía emocional y moral en el proceso de formación profesional. 


\section{Referencias bibliográficas.}

Arana, M., \& Batista, N. (1999). La educación en valores. Una propuesta pedagógica». Programa Educación en Valores. CTS. OEI.

Baquero, R. (2009). Zona de Desarrollo Próximo, sujeto y situación. El problema de unidades de análisis en psicología educativa. Actualidades investigativas en Educación, 1-25.

Bisquerra, R. (2016). Educación emocional. Documento inédito elaborado para las I Jornadas del Máster en Resolución de Conflictos.

Castellanos, D. (2002). Aprender y enseñar en la Escuela: una concepción desarrolladora. La Habana: Instituto Superior Pedagógico "Enrique José Varona".

Fariñas, G. (2009). El Enfoque Histórico Cultural para el estudio del desarrollo humano:para una praxis humanista. Actualidades Investigativas en Educacion, Vol-9, p-1-23.

Goleman, D. (1996). La inteligencia emocional en la empresa. (J. Vergara, Ed.) Buenos Aires.

Hernández González, M. G. (2015). El razonamiento moral, elemento de la competencia ética: dilemas reales e hipotéticos en la formación inicial del profesorado de Secundaria en México. Revista Complutense de Educación, 26(1), 183-201.

Ibarra Rosales, G. (2005). Ética y formación profesional integral. Universidad Autónoma Metropolitana Unidad Xochimilco, Distrito Federal. Obtenido de http://www.redalyc.org/articulo.oa?id?=34004303

Mertens, L. (1997). Competencia laboral: sistemas, surgimiento y modelos. Montevideo: CINTERFOR/OIT.

Montiel, F. J. (2018). La formación de la autonomía moral desde el preescolar. VARONA,Revista Científico-Metodológica,(No. 66). Obtenido de http://scielo.sld.cu/pdf/vrcm/n66/1992-8238-vrcm-66-e20.pdf

Moragues, D. (2006). Turismo y Cultura. Madrid, España: Agencia Española de Cooperación Internacional.

Morales, Á. H. (julio-diciembre de 2014). Competency of Tourist Animator: approaches form a French perspective. Universidad Autónoma de Tamaulipas. 
Nieva, J. A., \& Martínez, O. (2016). Una mirada sobre la formación docente. Revista Universidad y Sociedad., 8(4), p. 14-21.

Nouri, A. (2014). Dialogic Learning: a social conitive neuroscience view. International Journal of Cognitive Research in Science, Engineering and Education, 55-60.

Perrenoud, P. (2001). La formación de los docentes en el siglo XXI. p 511. Obtenido de 15 de junio2018, de http://www.colombiaaprende.edu.co/html/productos/1685/articles312338_APOYO6.pdf

Rico, P. (1990). ¿Cómo desarrollar en los alumnos las habilidades para el control y la valoración de su trabajo docente? . La Habana, Cuba: Editorial Pueblo y Educación.

Rodríguez Aguilera, Y. (2012). Diagnóstico de Valores Referenciales sobre Competencias de Calidad Personal en Empresas Turísticas. Universidad de La Habana . Trabajo de Diploma. Facultad de Turismo, La Habana, Cuba.

Sierra, R. (2008). La estrategia pedagógica, su diseño e implementación. La Habana, Cuba: Pueblo y Educación.

Tijero López, J. (2014). El perfeccionamiento de los procesos de comunicación organizacional de la Facultad de Turismo de la Universidad de la Habana: una propuesta. . Tesis de maestría, Facultad de Turismo de la Universidad de la Habana., Ciudad de la Habana.

Tünnermann, C. (2011). Educación universitaria para el siglo XXI. Análisis comparados (I). La educación universitaria necesaria para el siglo XXI. En L. Bonilla y F. López (Comp), 277-314. Caracas, Venezuela: Centro Internacional Miranda.

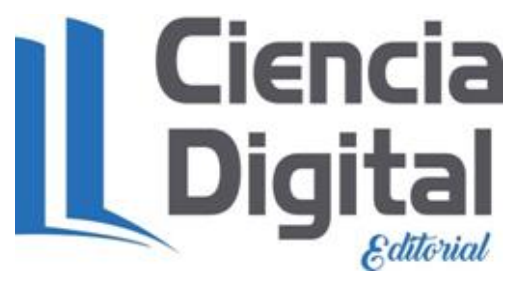


ISSN: 2600-5859

\section{Para citar el artículo indexado.}

Valdés Bencomo, Y., \& Martínez Chacón, O. (2020). Estrategia pedagógica para el desarrollo de la competencia de autonomía emocional y moral en los estudiantes de Turismo de la Universidad de La Habana. ConcienciaDigital, 3(1), 6-22. https://doi.org/10.33262/concienciadigital.v3i1.995

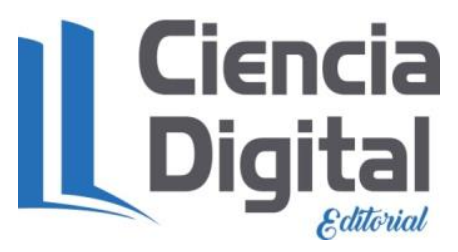

El artículo que se publica es de exclusiva responsabilidad de los autores y no necesariamente reflejan el pensamiento de la Revista Conciencia Digital.

El articulo queda en propiedad de la revista y, por tanto, su publicación parcial y/o total en otro medio tiene que ser autorizado por el director de la Revista Conciencia Digital.
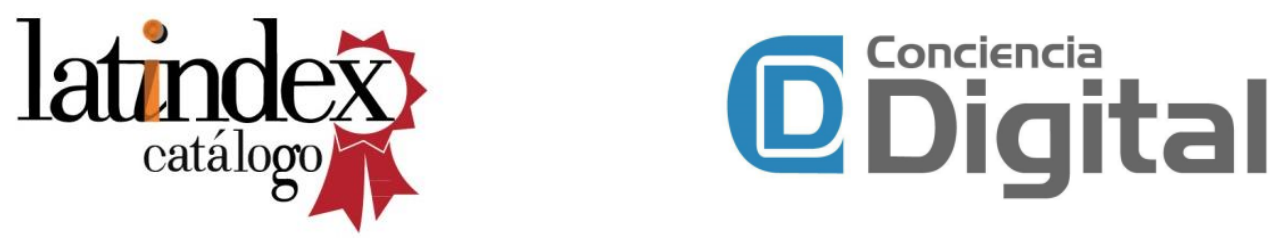